\title{
Hábitos y actitudes de los jóvenes ante las redes sociales: influencia del sexo, edad y clase social
}

\section{Social network practices and attitudes of young people: the influence of sex, age, and social class}

\author{
María-Cruz López-de-Ayala; Ricardo Vizcaíno-Laorga; Manuel Montes-Vozmediano
}

Cómo citar este artículo:

López-de-Ayala, María-Cruz; Vizcaíno-Laorga, Ricardo; Montes-Vozmediano, Manuel (2020). “Hábitos y actitudes de los jóvenes ante las redes sociales: influencia del sexo, edad y clase social". Profesional de la información, v. 29, n. 6, e290604.

https://doi.org/10.3145/epi.2020.nov.04

Artículo recibido el 18-05-2020

Aceptación definitiva: 02-09-2020

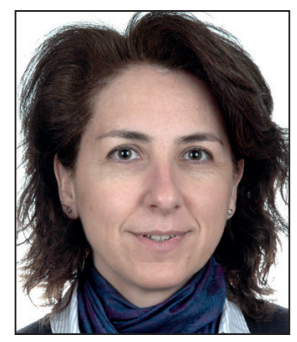

María-Cruz López-De-Ayala $\square$ https://orcid.org/0000-0002-6989-866X

Universidad Rey Juan Carlos

Facultad de Ciencias de la Comunicación

Camino del Molino, 5

29943 Fuenlabrada (Madrid), España

mariacruz.lopezdeayala@urjc.es

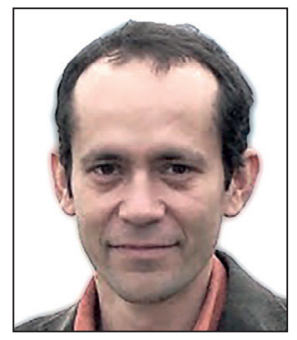

Ricardo Vizcaíno-Laorga

https://orcid.org/0000-0003-3164-8099

Universidad Rey Juan Carlos

Facultad de Ciencias de la Comunicación

Camino del Molino, 5

28942 Fuenlabrada (Madrid), España

ricardo.vizcaino@urjc.es

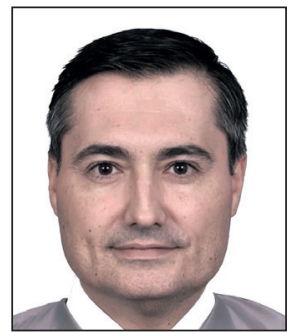

Manuel Montes-Vozmediano https://orcid.org/0000-0001-6624-8699

Universidad Rey Juan Carlos

Facultad de Ciencias de la Comunicación

Camino del Molino, 5

28942 Fuenlabrada (Madrid), España

manueljavier.montes@urjc.es

\section{Resumen}

En un contexto multiplataforma de uso generalizado y muy intensivo por parte de los jóvenes, estos presentan niveles de participación diferenciales según sus distintas motivaciones para participar. En este estudio se realiza un mapa de las prácticas más relevantes de los jóvenes en redes sociales, poniendo especial interés en identificar los usos participativos y estudiando sus actitudes hacia estos espacios que respaldan o limitan su comportamiento online; todo ello teniendo en cuenta aspectos como el sexo, la edad o la clase social familiar. La metodología cuantitativa del estudio analiza los resultados de una encuesta estadística representativa de los jóvenes de 17 a 24 años de la Comunidad de Madrid ( $N=533$ ). Los datos confirman el uso especialmente intensivo de los medios sociales que hacen los jóvenes, y que se orienta fundamentalmente hacia el entretenimiento y la interacción con su entorno más cercano. No obstante, estas cifras bajan drásticamente cuando se trata de acciones más participativas, con diferencias que destacan según la clase social. En conjunto, y de acuerdo con los resultados de un estudio factorial exploratorio, podemos identificar cuatro tipos de usos: informativo, participativo, sociabilidad cercana, y recreativo. Por otra parte, los jóvenes perciben las redes sociales, sobre todo, como espacios de información, para compartir opiniones y experiencias con la comunidad y de participación de la gente corriente, que les permite influir sobre la realidad, aunque también muestran cautela respecto a posibles consecuencias cuando se publican opiniones e información personal. La valoración que hacen de estas plataformas y sus repercusiones dependen del sexo, la edad y la clase social familiar, y también permiten predecir los tipos de usos, pero con efectos limitados. 


\title{
Palabras clave
}

Redes sociales; Medios sociales; Jóvenes; Participación; Actitudes; Hábitos; Género; Clase social; Entretenimiento; Ocio; Sociabilidad; Información.

\begin{abstract}
Young people show differential levels of participation in social networks linked to their different motivation to participate, even though their use is very high and intensive. In this study, a map of the most relevant social network practices of young people is constructed, with a particular focus on identifying participatory uses and studying their attitudes towards these spaces that support or limit their online behavior, while taking into account aspects such as sex, age, or family social class. The quantitative methodology applied in the current work analyzes the results of a representative statistical survey of young people aged 17 to 24 years in the Community of Madrid $(N=533)$. The results confirm the high and intensive use of social media by young people, mainly oriented towards entertainment and interaction with their closest environment. However, these figures drop drastically when it comes to more participatory actions, highlighting differences according to social class. Based on the results of an exploratory factor analysis, we identify four types of use: informative, participatory, close sociability, and recreational. On the other hand, young people perceive social networks especially as spaces for information, to share opinions and experiences with the community, and for the participation of the general public, which allows them to influence reality, although they also express caution regarding the possible consequences of publishing opinions and personal information. Their assessment of these platforms and their repercussions is linked to sex, age, and family social class, making it possible to predict the different types of use, albeit with limited effects.
\end{abstract}

\section{Keywords}

Social media; Social networks; Youth; Participation; Attitudes; Practices; Gender; Social class; Entertainment; Sociability; Information.

\section{Introducción}

Aunque existe un consenso sobre el uso generalizado y muy intenso por parte de los jóvenes de los medios sociales, estos ofrecen una amplia diversidad de usos de los que derivan diferentes oportunidades. Por un lado, las redes sociales brindan la capacidad de transcender, no solo el tiempo y el espacio, sino también la comunicación personal, accediendo a vías públicas de interacción que elevan el alcance de la expresión de las opiniones, quejas o demandas, más allá del entorno más cercano de familiares y amigos. No obstante, no todos los usuarios aprovechan tales ventajas de interacción para amplificar el alcance de sus posibilidades de expresión e influencia social. Aunque la comunicación en red rompe algunas barreras de participación, aún persisten limitaciones a este tipo de comportamiento, entre las que cabe mencionar la propia representación que los jóvenes hacen de estos espacios en base a sus propias experiencias de vida.

Por otra parte, según la perspectiva de la tercera brecha digital, aspectos tradicionales de la estratificación social enlazan con diversas formas de desigualdad digital -tipos de usos, habilidades y motivaciones (objeto de estudio de la llamada segunda brecha digital) - y, paralelamente, estos últimos revierten en diferentes beneficios sociales que reproducen las condiciones sociales de partida (Ragnedda; Muschert, 2018).

De acuerdo con lo anterior, el objetivo general de esta investigación es conocer los tipos de usos que los jóvenes hacen de las redes sociales, con especial atención a los participativos, e indagar las variables que inciden en este tipo de uso. En concreto, se analiza la influencia del sexo, la edad y la clase social sobre los tipos de usos y las representaciones de las redes sociales entre los jóvenes.

\section{Antecedentes y marco teórico}

En el mundo 4.500 millones de personas usan internet una media de 6:43 horas diarias. El 84\% de ellas utiliza redes sociales (Datareportal, 2020), y es una tendencia en alza (Statista, 2019). Pero el consumo mundial no es homogéneo. Brasil, Filipinas o Suráfrica superan las 9 horas de consumo de internet, mientras Alemania, Japón y Países Bajos le dedican menos de 5 horas. España y Reino Unido tienen un consumo idéntico (5:41 horas), mientras Estados Unidos y Portugal se sitúan muy próximos al consumo medio (Datareportal, 2020). El consumo de internet es un fenómeno diverso. Y las causas de dicha diversidad, son complejas.

Investigar hábitos o usos (no el mero consumo) plantea retos aún mayores con los que se hace necesario tomar decisiones. La propia tipología de clasificaciones sobre usos y usuarios que recoge la bibliografía es ya de por sí muy variada y depende del método, teoría y el punto de interés en el que se centra cada estudio. Algunas de ellas han sido criticadas. Blank y Groselj (2014), después de revisar clasificaciones previas, plantean un "espacio de uso" de Internet en el que cada usuario podría situarse al menos según tres dimensiones: cantidad (horas de consumo), variedad (una red social o muchas) y tipos de usos de Internet (por ejemplo, para informarse, participar políticamente o comunicarse con familiares). Así los usuarios quedan ubicados y caracterizados en ese espacio cartesiano. Estos autores cuestionan otras tipologías que mezclan las tres dimensiones, como hablar de "Usuarios esporádicos" (dimensión cantidad), "Usuarios de redes sociales y entretenimiento" y "Usuarios instrumentales" (dimensión tipos de usos) y "Usuarios avanzados" (dimensión variedad) (Brandtzæg; Heim; Karahasanović, 2011; Borg; Smith, 2018). 
El propio "uso" no es el único eslabón de la cadena. Históricamente se han investigado tres aspectos: el acceso, la adopción y el uso o hábitos en Internet. El acceso y la adopción fueron temas recurrentes décadas atrás (Sathye, 1999; Gloy; Akridge, 2000; Van-Rompaey; Roe; Struys, 2002; Awoleye; Siyanbola; Oladipo, 2008; Hill; Beynon-Davies; Williams, 2008; Birba; Diagne, 2012; Nasri; Charfeddine, 2012), pero hoy son menos habituales y el punto de atención se encuentra fundamentalmente en el uso de Internet desde diferentes enfoques, bien en estudios descriptivos y de tendencia, diagnósticos, o explicativos. Y en todos ellos las variables posibles de análisis también pueden ser diferentes: edad, sexo, situación económica, temas o propósitos de uso (uso profesional, familiar, entretenimiento), tipos de públicos (desde profesiones hasta discapacitados), técnicas de acceso, plataformas o capacitaciones personales, entre otras.

La bibliografía demuestra que las variables sociodemográficas siguen resultando útiles como elementos predictores del uso general de Internet y también del uso de redes sociales, pero que continúan siendo un punto de desigualdad (Gonzales; Yan, 2020). Pero si la propia definición de red social ya resultó controvertida desde sus orígenes (Ros-Martín, 2009; Kaplan; Haenlein, 2010), el hecho mismo de centrar el estudio de usos en el caso concreto de redes sociales hace que surjan factores que pueden interactuar. Diversos autores incluyen la soledad como un posible elemento a considerar en el uso de las redes sociales (Khalaila; Vitman-Schorr, 2017; Nowland; Necka; Cacioppo, 2017). Symons et al. (2019) incluyen la mediación parental como un elemento modulante de los usos de Internet que hacen los hijos. Por tanto, las investigaciones muy focalizadas podrían no ser tan simplistas.

Pero las variables demográficas, de una u otra forma, siguen siendo el punto en común de estos estudios. A través de estas variables se han tratado de buscar patrones de uso. Blank y Groselj (2014) utilizaron siete variables demográficas (edad, sexo, urbano/rural, etnia, nivel educativo, nivel socioeconómico y estado civil) como predictores de uso en diez diferentes actividades (entretenimiento, comercio, búsqueda de información, relaciones sociales, email, blog, producción de información, medios convencionales, deberes del colegio y vicios). Después de aplicar un análisis de regresión, los autores sugieren que las personas con determinadas características estarían relacionadas con cada tipo de actividad. Y que cada una de las diez actividades puede considerarse una dimensión separada. Edad y nivel educativo fueron las variables más importantes, seguidas del sexo. Así los jóvenes con mayor nivel de educación y los usuarios hombres tendrán mayor probabilidad de hacer un uso más frecuente y más variado de Internet. Büchi, Just y Latzer (2016) también comprobaron que la edad predice cuatro tipos de uso (interacción social, búsqueda de información, entretenimiento y comercio electrónico), el sexo explicaría parcialmente el uso social de internet y el nivel educativo lo haría para la búsqueda de información y el comercio electrónico. Correa (2015) encontró que la edad, el género y el nivel educativo influía en un mejor uso de las redes sociales. Pero las influencias de las variables demográficas varían con el tiempo y el contexto, lo que exige estudios de seguimiento que actualicen los datos y confirmen o modifiquen las tendencias previamente observadas tanto en contextos locales (Sánchez-Martínez; Otero, 2010) como en macro contextos: mientras en Europa occidental la utilización de las redes sociales es prácticamente idéntica por sexo (49\% mujeres, 51\% hombres), en el sur de Asia las diferencias son muy acusadas (24\% y $76 \%$, según Datareportal, 2020).

Por otra parte, las propias redes sociales pueden ser variables predictoras. Kircaburun (2016) encontró relación indirecta entre la adicción a las redes sociales y la depresión y Shensa et al. (2017) sugieren que es el cómo (y no el cuánto) lo que establecería esa relación. García-Jiménez, López-de-Ayala y Catalina-García (2013) observaron relación entre el uso de redes sociales y el tiempo de uso de Internet. Liu, Kirschner y Karpinski (2017) comprobaron que las redes sociales y el rendimiento académico de jóvenes y adolescentes podrían estar conectados, y es que los jóvenes son conscientes del tiempo que "pierden" en ellas (García-Jiménez; López-de-Ayala; Montes-Vozmediano, 2020). Por su parte, Knight-McCord et al. (2016) afirman que la presencia de determinadas características de las redes sociales (como la posibilidad de publicar fotos o videos) son motivaciones para elegirlas frente a otras, así como el creciente consumo audiovisual, que en el caso de los jóvenes no solo se circunscribe al entretenimiento o al ocio (Pastor-Ruiz; Martín-Nieto; Montes-Vozmediano, 2019) sino también a contenidos formativos de los temas de su interés y presentados con un enfoque positivo (García-Jiménez; Montes-Vozmediano, 2020).

En cuanto a los estudios motivacionales sobre uso y participación social en redes, López-González y Anaya-Rodríguez (2016) constataron "el poco interés hacia los problemas sociales y ciudadanos, defensa de derechos humanos y políticos", en favor de otras temáticas como el "ocio, diversión y entretenimiento, así como por aspectos educativo/académicos y artísticos/culturales", lo que coincide con otros estudios en los que el uso de las redes sociales se centraría fundamentalmente en las relaciones de amistad y familia, las profesionales o la búsqueda de información o el entretenimiento (Masip et al., 2015). Una revisión exhaustiva sobre la participación social de los jóvenes puede encontrarse en Catalina-García, Martín-Nieto y López-de-Ayala (2018) quienes concluyen que las nuevas tecnologías aportan prácticas diferentes de participación, pero que dicha participación online parece tener mucho que ver con la actitud de cada persona en la esfera real o física. En este sentido, en las redes sociales se pueden encontrar diversas posturas: desde el clicktivismo de bajo esfuerzo, consistente en "participar" mediante un like o un compartir (Karpf, 2010; Drumbl, 2012; García-Estévez, 2018), a la participación activa. La bibliografía no ha aclarado hasta qué punto un tipo de participación (online) influye realmente en la otra (presencial) o viceversa, pero sí parecen mostrarse relacionadas a la vez que ser diferentes. Que los medios sociales son instrumentos de las organizaciones parece un hecho constatado. Y que no motivan la participación ni la interacción con el ciudadano, también (Catalina-García; Martín-Nieto; López-de-Ayala, 2018; Vizcaíno-Laorga; Catalina-García; López-de-Ayala-López, 2019). 


\section{Objetivos}

El objetivo general de este artículo es conocer los hábitos de uso de las redes sociales por los jóvenes, con especial atención a los usos participativos, e indagando en las variables que inciden en los tipos de utilización. De acuerdo con este propósito general, los objetivos secundarios son:

- conocer los usos que los jóvenes hacen de las redes sociales;

- generar una tipología de usos de internet entre los jóvenes, con especial interés en identificar una modalidad de uso participativo que pueda diferenciarse de otras formas;

- desvelar cómo el sexo, la edad y la clase social inciden en cada modalidad;

- conocer las actitudes que respaldan los tipos de utilización de las redes sociales, analizando la influencia de la sexo, edad y clase social; y, por último,

- examinar las relaciones entre las representaciones de las redes sociales y los tipos de usos.

\section{Metodología}

\subsection{Participantes}

La base del estudio es una encuesta a una muestra representativa de los jóvenes de 17 a 24 años de la Comunidad de Madrid $^{1}$. Se aplicó un muestreo bietápico, estratificado por conglomerados, con selección de las unidades primarias de muestreo (municipios y secciones censales) de forma aleatoria proporcionada y de las unidades últimas (individuos) por rutas aleatorias y cuotas de sexo y edad. La base muestral se diseñó sobre los datos correspondientes a la población interpolada por edad, para cada sexo con fecha de referencia de 1 de enero de 2019 para la Comunidad de Madrid (INE, 2019). El error muestral queda fijado en el \pm 4 , para datos globales, para $p=q=0,5$, con un nivel de confianza del $95 \%$.

La muestra estaba formada por 533 entrevistados ( $51,2 \%$ de chicos y $48,7 \%$ de chicas; y $M_{\text {edad }}=20,52$ años, $D S=2,309$ ). En el análisis, se incluyeron pesos para ajustar una leve desviación observada entre la distribución de la muestra por edad y sexo respecto a la que presenta el universo de jóvenes.

\subsection{Variables e instrumentos}

Para la recogida de información se utilizó un cuestionario de elaboración propia, en el que se les preguntó, entre otras cuestiones, sobre la frecuencia de uso diario general de redes sociales (escala de respuesta desde "No lo uso" hasta "Continuamente"; para el análisis multivariante se codificó de 1 a 6 eliminando "No lo uso"), la frecuencia de uso de diferentes tipos de actividades en las mismas (codificado de 1 a 6 , desde "Nunca o casi nunca" hasta "Continuamente") y su grado de acuerdo con afirmaciones que reflejan diferentes creencias sobre las redes sociales (opciones de respuesta desde $1=$ "Nada de acuerdo" hasta $5=$ "Totalmente de acuerdo").

Las variables sociodemográficas consideradas fueron sexo ( $1=$ hombre; $2=$ mujer), edad y clase social $(5,2 \%$ se clasificó como de clase baja; $21 \%$ de clase media-baja; $44,8 \%$ media-media; $16 \%$ media-alta; y $13 \%$ alta). Esta última ha sido construida en torno a dos variables: ocupación y nivel educativo del padre, asumiendo que es quien más ingresos aporta; salvo en aquellos casos en los que el padre esté en paro, sea pensionista, etc., que se ha considerado a la madre.

\subsection{Procedimiento}

La administración de las encuestas se realizó entre el 17 de junio y el 4 de julio de 2019, mediante el Sistema CAPI (entrevista personal asistida con ordenador) en el domicilio de los encuestados. La cumplimentación de las encuestas llevó alrededor de unos 25 minutos.

\subsection{Análisis de datos}

Los datos obtenidos de la encuesta han sido analizados con el programa estadístico SPSS, $v .26$ y el nivel de validez estadística se ha establecido para el valor $p<.05$. Con el fin de obtener un modelo reducido de tipos de uso de medios sociales entre los jóvenes, se ha realizado un análisis factorial exploratorio de componente principales. Este método estadístico describe la variabilidad entre variables correlacionadas observadas y crea un modelo como combinación de potenciales factores, evitando redundancias.

Para las variables dependientes ordinales se ha aplicado la prueba t-test (si se cumple el criterio de normalidad) y el test no paramétrico de U de Mann-Whitney para comparar dos muestras independientes, adecuado para ver si las diferencias de medias de una variable ordinal o que no se ajustan a la curva normal se pueden inferir al conjunto de la población.

Para analizar las relaciones entre variables ordinales y variables cuantitativas se ha utilizado el coeficiente de correlación de Pearson $(r)$ y de Spearman $(r)$, según la distribución de los datos se ajustaran o no a la curva normal. Asimismo, como en las tablas de contingencia se ha observado que la clase social no muestra efectos lineales sobre las variables analizadas, se ha tratado como una variable nominal y se ha aplicado Anova de un solo factor y el gamma de Goodman-Kruskal y test de Kruskal-Wallis, según si se cumplen los supuestos o no de normalidad; posteriormente, se ha realizado un análisis post hoc, usando la corrección Bonferroni, para comparar los resultados dos a dos de las categorías de la variable independiente, con el objetivo de descubrir las categorías que mostraban diferencias significativas entre ellas, calculando el tamaño del efecto (eta al cuadrado, $\eta^{2}$ ). 


\section{Resultados}

\subsection{Datos generales de uso}

En primer lugar se observa una utilización muy intensiva de las redes sociales por parte de los jóvenes, con un uso mayoritario diario: $94 \%$ accede a ellas todos los días y $83,5 \%$ lo hace continuamente o varias veces al día. El test de U-Mann-Whitney indica que las diferencias por sexo son significativas para el conjunto de la población $(Z=-2,067, p<.05)$, con una frecuencia media de conexión ligeramente superior entre las mujeres $(M=5.37)$ frente a los hombres $(M=5.27$; donde 1 es con menos frecuencia y 6 es continuamente), y el coeficiente de correlación de Spearman indica que los más jóvenes se conectan

Tabla 1. Frecuencia de uso diario de redes sociales (\%)

\begin{tabular}{|l|c|}
\hline Continuamente & 52,9 \\
\hline Varias veces al día & 30,6 \\
\hline Un rato todos los días & 10,7 \\
\hline 3-4 días a la semana & 3,0 \\
\hline Con menos frecuencia & 1,3 \\
\hline No lo uso & 1,5 \\
\hline Base total & 533 \\
\hline
\end{tabular}
con más frecuencia $\left(r_{s}=.141\right.$, para $\left.p<.01\right)$. Sin embargo, gamma de Goodman-Kruskal ( $(\nu$ ) y el test de Kruskal-Wallis no muestran una asociación significativa entre clase social y frecuencia de uso.

\subsection{Tipos de usos de redes sociales entre los jóvenes}

Los datos recabados confirman el uso preferente hacia la interacción con el entorno más cercano y el entretenimiento. De manera que conversar con amigos y familiares, ver sus fotos y vídeos y vídeos y música son las actividades realizadas mayoritariamente por los usuarios de estas plataformas: alrededor de 70\% de los jóvenes realizan estas acciones conti-

Tabla 2. Frecuencia con la que los jóvenes realizan actividades en redes sociales. En porcentajes horizontales

\begin{tabular}{|c|c|c|c|c|c|c|}
\hline Usos de redes sociales & $\begin{array}{l}\text { Continua- } \\
\text { mente (6) }\end{array}$ & $\begin{array}{c}\text { Todos o casi } \\
\text { todos los días } \\
\text { (5) }\end{array}$ & $\begin{array}{c}304 \text { veces en } \\
\text { semana } \\
\text { (4) }\end{array}$ & $\begin{array}{c}1 \text { o } 2 \text { veces en } \\
\text { semana } \\
\text { (3) }\end{array}$ & $\begin{array}{c}\text { Alguna vez al } \\
\text { mes } \\
(2)\end{array}$ & $\begin{array}{c}\text { Nunca o casi } \\
\text { nunca } \\
(1)\end{array}$ \\
\hline $\begin{array}{l}\text { Publicar/colgar contenidos perso- } \\
\text { nales en tu perfil }\end{array}$ & 27,9 & 27,5 & 17,2 & 12,0 & 10,1 & 5,2 \\
\hline $\begin{array}{l}\text { Ver vídeos o fotos de amigos o } \\
\text { familiares }\end{array}$ & 33,7 & 35,8 & 17,8 & 9,0 & 2,8 & 1,0 \\
\hline Hablar con amigos & 45,6 & 29,7 & 11,4 & 7,8 & 3,7 & 1,9 \\
\hline Hablar con familiares & 30,9 & 32,4 & 16,0 & 9,2 & 5,9 & 5,7 \\
\hline Ver videos y música & 34,9 & 33,5 & 18,6 & 9,4 & 2,9 & 0,6 \\
\hline $\begin{array}{l}\text { Buscar contenido deportivo: } \\
\text { deportes y deportistas }\end{array}$ & 12,4 & 16,3 & 21,3 & 14,3 & 14,8 & 20,9 \\
\hline $\begin{array}{l}\text { Buscar información sobre ocio: } \\
\text { cine, libros, conciertos... }\end{array}$ & 11,1 & 17,3 & 26,0 & 20,7 & 13,4 & 11,5 \\
\hline $\begin{array}{l}\text { Buscar información comercial } \\
\text { (marcas, productos, empresas) }\end{array}$ & 11,1 & 17,2 & 20,7 & 18,0 & 15,0 & 18,1 \\
\hline $\begin{array}{l}\text { Buscar información sobre series, } \\
\text { películas o programas de televisión }\end{array}$ & 11,9 & 21,2 & 23,0 & 19,9 & 12,0 & 12,0 \\
\hline $\begin{array}{l}\text { Buscar información sobre famosos: } \\
\text { actores o cantantes, deportes... }\end{array}$ & 9,0 & 15,8 & 22,4 & 21,6 & 16,1 & 15,1 \\
\hline $\begin{array}{l}\text { Seguir a profesionales de tu cam- } \\
\text { po de trabajo o estudio }\end{array}$ & 9,9 & 17,3 & 15,1 & 16,2 & 14,5 & 27,0 \\
\hline $\begin{array}{l}\text { Buscar información salud, dietas/ } \\
\text { nutrición y bienestar }\end{array}$ & 9,5 & 12,0 & 17,0 & 16,5 & 15,8 & 29,2 \\
\hline $\begin{array}{l}\text { Buscar información belleza, moda } \\
\text { y estilismo }\end{array}$ & 10,6 & 15,9 & 18,5 & 15,3 & 13,5 & 26,2 \\
\hline Buscar contenidos divertidos & 14,5 & 28,1 & 25,0 & 15,2 & 8,8 & 8,4 \\
\hline $\begin{array}{l}\text { Seguir a tus actores, cantantes, } \\
\text { deportistas, influencers favoritos }\end{array}$ & 10,9 & 17,3 & 21,6 & 20,0 & 13,2 & 17,1 \\
\hline Jugar online & 9,7 & 11,8 & 10,2 & 11,2 & 9,8 & 47,3 \\
\hline Comprar y vender & 4,2 & 8,2 & 7,7 & 11,9 & 30,1 & 38,0 \\
\hline $\begin{array}{l}\text { Publicar opiniones sobre temas } \\
\text { sociales o políticos }\end{array}$ & 10,1 & 12,9 & 8,3 & 12,3 & 12,8 & 43,6 \\
\hline $\begin{array}{l}\text { Publicar críticas o quejas en un } \\
\text { perfil público }\end{array}$ & 11,2 & 11,5 & 9,9 & 10,4 & 14,1 & 43,0 \\
\hline $\begin{array}{l}\text { Compartir o recomendar sitios y } \\
\text { enlaces a otros }\end{array}$ & 12,3 & 14,1 & 13,3 & 15,9 & 14,7 & 29,7 \\
\hline
\end{tabular}


nuamente o todos o casi todos los días. A continuación, se sitúan publicar contenidos en el perfil (55\%) y consultar contenidos divertidos (43\%). Sin embargo, las cifras bajan drásticamente cuando nos centramos en usos más participativos, como son publicar opiniones sobre temas sociales o políticos y emitir críticas o quejas en un perfil público: alrededor del $20 \%$. Por último, comprar y vender se sitúa al final de las opciones de tareas realizadas con más asiduidad por los jóvenes en estas plataformas. Más significativo resulta que estas actividades más participativas, junto con jugar online, no se realicen nunca o casi nunca por un porcentaje muy importante de los entrevistados (más del 40\%), donde se sitúa la moda.

A continuación se han sometido los veinte items relativos a los usos en redes sociales a un análisis factorial basado en componentes principales, con vistas a desvelar la estructura latente subyacente del uso de redes sociales entre los adolescentes jóvenes, reduciendo el número de dimensiones y facilitando el análisis y la interpretación. Para extraer los factores, se seleccionaron los eigenvalues superiores a uno. Después de varias pruebas, se extrajeron dos items del análisis: "buscar contenidos divertidos", en la medida que mantenía una puntuación inferior a 0.5 en el análisis de comunalidades; y "comprar y vender" que mostró unas correlaciones inferiores a 0.5 con el resto de variables incluidas en el análisis factorial.

El modelo final proporcionado por el programa mostró cuatro dimensiones o factores que sintetizaban la información de los dieciocho items y que explicaban el 64,72\% de la varianza. El análisis mostró un ajuste de la muestra de 0.886 (Kaiser-Meyer-Olkin) y resulta significativo al nivel .000 (prueba de esfericidad de Bartlett), lo que indica que el modelo de correlaciones obtenidas no se debe al azar. Para mejorar la interpretación de los resultados se ha procedido a aplicar una rotación varimax de los factores obtenidos. De este modo, se puede delimitar más claramente los atributos que definen los cuatro factores obtenidos, e interpretarlos siguiendo el criterio de seleccionar puntuaciones superiores a 0.5 . Los resultados aparecen reflejados en la tabla 3.

Tabla 3. Dimensiones de uso de redes sociales. Matriz de componentes rotado

\begin{tabular}{|c|c|c|c|c|}
\hline \multirow{2}{*}{ Usos de redes sociales } & \multicolumn{4}{|c|}{ Componentes } \\
\hline & 1 & 2 & 3 & 4 \\
\hline Publicar/colgar contenidos personales en tu perfil & .143 & .414 & .584 & .089 \\
\hline Ver vídeos o fotos de amigos o familiares & .098 & .105 & .767 & .113 \\
\hline Hablar con amigos & .080 & -.030 & .814 & -.033 \\
\hline Hablar con familiares & .194 & .041 & .710 & -.150 \\
\hline Ver videos y música & .145 & .125 & .706 & .187 \\
\hline Buscar contenido deportivo: deportes y deportistas & .298 & .257 & .136 & .692 \\
\hline Buscar información sobre ocio: cine, libros, conciertos, espectáculos, eventos fin de semana & .725 & .123 & .110 & .263 \\
\hline Buscar información comercial (marcas, productos, empresas) & .689 & .274 & .136 & .243 \\
\hline Buscar información sobre series, películas o programas de televisión & .751 & .180 & .141 & .213 \\
\hline Buscar información sobre famosos: actores o cantantes, deportes, fútbol, influencers... & .708 & .095 & .066 & .301 \\
\hline Seguir a profesionales de tu campo de trabajo o estudio & .640 & .350 & .168 & .175 \\
\hline Buscar información salud, dietas/nutrición y bienestar & .663 & .449 & .149 & -.100 \\
\hline Buscar información belleza, moda y estilismo & .748 & .229 & .151 & -.317 \\
\hline Seguir a tus actores, cantantes, deportistas, influencers favoritos & .593 & .155 & .220 & .274 \\
\hline Jugar online & .220 & -.002 & -.014 & .739 \\
\hline Publicar opiniones sobre temas sociales o políticos & .239 & .844 & .077 & .133 \\
\hline Publicar críticas o quejas en un perfil público & .286 & .875 & .090 & .049 \\
\hline Compartir o recomendar sitios y enlaces a otros & .290 & .809 & .151 & .074 \\
\hline Eigenvalues & 6.932 & 2.105 & 1.501 & 1.114 \\
\hline Varianza explicada & 38.503 & 11.693 & 8.337 & 6.189 \\
\hline \multicolumn{5}{|c|}{ Método de extracción: análisis de componentes principales. Método de rotación: Varimax con normalización Kaiser. } \\
\hline \multicolumn{5}{|l|}{ La rotación ha convergido en 6 iteraciones. } \\
\hline En negrita los items que puntúan por encima de 0.5 en cada factor. & & & & \\
\hline
\end{tabular}

A partir del análisis realizado se han podido extraer los siguientes factores o dimensiones en los usos de redes sociales entre los jóvenes:

Factor 1. Usos informativos. Incluye cargas superiores a 0.5 para los items: "Buscar información sobre ocio"; "Buscar información comercial"; "Buscar información sobre series, películas o programas de televisión"; "Buscar información sobre famosos; Seguir a profesionales de tu campo de trabajo o estudio"; "Buscar información salud, dietas/nutrición y bienestar"; "Buscar información belleza, moda y estilismo"; y "Seguir a tus actores, cantantes, deportistas, influencers favoritos". 
Factor 2. Usos participativos. Con cargas fuertes de los items: "Publicar opiniones sobre temas sociales o políticos"; "Publicar críticas o quejas en un perfil público"; y "Compartir o recomendar sitios y enlaces a otros".

Factor 3. Usos de socialización cercana. Contempla items vinculados con la sociabilidad cercana y ocio audiovisual con cargas elevadas: "Publicar/colgar contenidos personales en tu perfil"; "Ver vídeos o fotos de amigos o familiares"; "Hablar con amigos"; "Hablar con familiares"; y "Ver vídeos y música".

Factor 4. Usos recreativos. Incluye items con cargas superiores a 0.5 relativos a contenidos de tipo recreativo: "Jugar online" y "Buscar contenido deportivo".

\subsection{Influencia del sexo, edad y clase social}

Antes de proceder a los análisis, examinamos si las nuevas variables siguen una distribución normal con el objetivo de determinar qué tipo de contrastes vamos a aplicar. El test de Kolmogorov-Smirnov indica que únicamente el uso informativo cumple el supuesto de normalidad.

Con respecto al modelo de uso informativo, la prueba de hipótesis t-test indica que existen diferencias significativas entre las medias de puntuación por sexo, con una mayor tendencia de las mujeres a hacer este tipo de uso $(M=0,14$, $D S=0,64)$ frente a los hombres $(M=-0,13, D S=0,59, t(518)=-3.041, p<.01)$. Pero las diferencias no son significativas ni para la edad, ni para la clase social.

Centrando la atención en el modelo de uso participativo de redes sociales, la prueba no paramétrica de Mann-Whitney muestra que no existe una relación significativa con el sexo. Sin embargo, el coeficiente de correlación de Spearman sugiere que conforme aumenta la edad también lo hace el uso participativo $\left(r=.131^{* *}\right)$, aunque el grado de asociación es débil. En relación con la clase social, el gamma de Goodman-Kruskal señala una asociación positiva significativa débil con este tipo de uso $(\gamma=.156, p<.01)$. Y el test de Kruskal-Wallis, adecuado para examinar la relación entre variables cuantitativas y variables nominales de múltiples particiones, permitió corroborar esta asociación $\chi^{2}(4, N=520$, estadístico de prueba $=22.16$ y $p<.01)$, con un efecto moderado $\left(\eta^{2}=.042\right)$. Un test post-hoc usando la corrección Bonferroni apunta a que la clase media baja tiende a hacer un uso menos participativo con respecto a la clase media-media, la clase alta y la clase media-alta $(p<.01)$; al igual que la clase media-media con respecto a la clase media alta $(p<.05)$.

Por otra parte, el sexo $(\mathrm{Z}=-3,564 p<.001)$ y la edad $\left(r_{\mathrm{s}}=-.136^{* *}\right)$ muestran diferencias significativas en la tendencia a hacer usos de socialización cercana; con una mayor predilección de las mujeres $(M=0,14)$ frente a los hombres $(M=-0,14)$ y los más jóvenes por esta modalidad. El gamma de Goodman-Kruskal también muestra diferencias significativas estadísticamente por clase social $(p<.05)$, pero insignificante sociológicamente $(-.087)$ y que se pone de manifiesto en la ausencia de significación estadística cuando aplicamos el test de Kruskal-Wallis.

Por último, en relación con los usos recreativos, únicamente el sexo mantiene una relación estadísticamente significativa a favor de los varones $(M=0,48)$ frente a las mujeres $(M=-0,50, Z=-11,541, p<001)$, en tanto que ni la edad (rho de Spearman) ni la clase social (gamma de Goodman-Kruskal) muestran diferencias significativas estadísticamente.

\subsection{Opiniones sobre redes sociales}

En cuanto a las representaciones de las redes sociales, los jóvenes las perciben sobre todo como espacios de información (83,4\% están totalmente y bastante de acuerdo), controladas por intereses económicos y/o políticos (83,4\%), que pueden tener consecuencias negativas respecto a las opiniones que se suben $(83,2 \%)$, que permiten que la gente corriente pueda influir en asuntos públicos $(82,3 \%)$ y donde la gente solo muestra su cara más favorable $(81,3 \%)$. Además, se muestran temerosos del uso que se pueda hacer de su información personal $(79,4 \%)$; y, mientras que perciben que estas plataformas permiten que la gente corriente pueda defender sus intereses $(78,4 \%)$, paralelamente señalan que no permiten cambiar nada $(78,4 \%)$. Por tanto, las representaciones de estas plataformas oscilan entre el optimismo por las oportunidades que ofrecen de información, de expresión de intereses personales y de influencia en asuntos públicos, el escepticismo sobre su capacidad de poder influir en asuntos públicos y los riesgos que ocultan de represalias en cuanto a la expresión de opiniones y mal uso de la información personal que suben los usuarios.

Atendiendo a las diferencias de opiniones sobre las redes sociales por sexo, el test de U de Mann-Whitney muestra diferencias de medias significativas para las siguientes afirmaciones: "La gente miente mucho en redes sociales" $\left.M_{\text {hombre }}=4.07 ; M_{\text {mujer }}=4.2\right)(p<.05)$; "Las redes sociales facilitan que la gente corriente pueda defender sus intereses" $\left(M_{\text {hombre }}=3.78 ; M_{\text {mujer }}=3.96\right)(p<.05)$; "En redes se pueden encontrar otras ideas y puntos de vista muy interesante" $\left(M_{\text {hombre }}=3.97 ; M_{\text {mujer }}=4.15\right)(p<.05)$; "Hay que tener mucho cuidado con la información personal que se sube a redes sociales" $\left(M_{\text {hombre }}=4.17 ; M_{\text {mujer }}=4.3\right)(p<.05)$; "La gente solo muestra su cara más favorable en las redes sociales" $\left(M_{\text {hombre }}=4.08 ; M_{\text {mujer }}=4.2\right)(p<.05)$; "Las redes sociales permiten que la gente pueda mostrar sus quejas o malestar sobre las cosas que no les gusta" $\left(M_{\text {hombre }}=3.95 ; M_{\text {mujer }}=4.12\right)(p<.05)$. De nuevo encontramos que las diferencias significativas en las opiniones sobre redes sociales entre hombres y mujeres son muy débiles. 
Tabla 4. Opiniones sobre las redes sociales. En porcentajes horizontales

\begin{tabular}{|c|c|c|c|c|c|}
\hline Opiniones & $\begin{array}{c}\text { Total- } \\
\text { mente de } \\
\text { acuerdo }\end{array}$ & $\begin{array}{l}\text { Bastante } \\
\text { de } \\
\text { acuerdo }\end{array}$ & $\begin{array}{c}\mathrm{Ni} \\
\text { acuerdo/ } \\
\text { ni des- } \\
\text { acuerdo }\end{array}$ & $\begin{array}{l}\text { Poco de } \\
\text { acuerdo }\end{array}$ & $\begin{array}{l}\text { Nada de } \\
\text { acuerdo }\end{array}$ \\
\hline Las redes sociales favorecen que todos estemos mejor informados & 37,3 & 46,1 & 10,9 & 2,7 & 3 \\
\hline $\begin{array}{l}\text { A través de las redes la gente puede compartir sus opiniones y experien- } \\
\text { cias con la comunidad }\end{array}$ & 39,5 & 38,7 & 17 & 4,4 & 0,3 \\
\hline La gente solo muestra su cara más favorable en las redes sociales & 39,3 & 42 & 15,6 & 1,7 & 1,4 \\
\hline $\begin{array}{l}\text { Hay que tener mucho cuidado con las opiniones que se suben a las redes } \\
\text { sociales porque puede tener consecuencias negativas }\end{array}$ & 29,2 & 54 & 13,5 & 2,5 & 0,8 \\
\hline $\begin{array}{l}\text { Las redes sociales permiten una mayor participación de la gente corriente, } \\
\text { influyendo en las opiniones de otros }\end{array}$ & 28,9 & 44 & 20,5 & 5,4 & 1,3 \\
\hline Permiten tener un mayor conocimiento sobre la realidad que nos rodea & 24,5 & 37,5 & 28,3 & 6,3 & 3,3 \\
\hline En las redes sociales cualquiera puede opinar sin miedo a tener represalias & 40,4 & 37,9 & 17,5 & 3 & 1,3 \\
\hline La gente miente mucho en redes sociales & 32,2 & 39,4 & 19,2 & 6,8 & 2,4 \\
\hline Hoy en día estás totalmente controlado en las redes sociales & 25,7 & 42,1 & 26,5 & 4,4 & 1,4 \\
\hline $\begin{array}{l}\text { Las redes sociales facilitan que la gente corriente pueda defender sus } \\
\text { intereses }\end{array}$ & 32,1 & 46,3 & 17,5 & 3,3 & 0,7 \\
\hline $\begin{array}{l}\text { En redes se pueden encontrar otras ideas y puntos de vista muy intere- } \\
\text { santes }\end{array}$ & 22,7 & 31,3 & 28,1 & 13,1 & 4,9 \\
\hline Las redes sociales te aíslan de la vida real & 23,5 & 35,9 & 28 & 8,7 & 3,8 \\
\hline $\begin{array}{l}\text { Las redes sociales son clave para que podamos tomar decisiones en asun- } \\
\text { tos sociales y políticos }\end{array}$ & 24 & 42,6 & 24,4 & 6,3 & 2,6 \\
\hline $\begin{array}{l}\text { Las redes sociales proporcionan a la gente corriente más capacidad de } \\
\text { influir en asuntos públicos }\end{array}$ & 44,7 & 37,6 & 14,3 & 2,6 & 0,7 \\
\hline $\begin{array}{l}\text { Hay que tener mucho cuidado con la información personal que se sube a } \\
\text { redes sociales }\end{array}$ & 41 & 38,4 & 15,3 & 3,9 & 1,3 \\
\hline La gente solo muestra su cara más favorable en las redes sociales & 26,8 & 45,1 & 21,5 & 5 & 1,6 \\
\hline $\begin{array}{l}\text { Las redes sociales hacen posible que todos podamos participar en el } \\
\text { debate de temas públicos }\end{array}$ & 22,4 & 32,7 & 31,3 & 9,7 & 3,9 \\
\hline $\begin{array}{l}\text { Los comentarios que la gente corriente sube a las redes sociales no sirven } \\
\text { para cambiar nada }\end{array}$ & 30,9 & 47,5 & 16,3 & 4,6 & 0,6 \\
\hline $\begin{array}{l}\text { Las redes sociales son herramientas controladas por intereses económicos } \\
\text { y/o políticos }\end{array}$ & 37,3 & 46,1 & 10,9 & 2,7 & 3 \\
\hline $\begin{array}{l}\text { Las redes sociales permiten que la gente pueda mostrar sus quejas o } \\
\text { malestar sobre las cosas que no les gusta }\end{array}$ & 39,5 & 38,7 & 17 & 4,4 & 0,3 \\
\hline
\end{tabular}

Respecto a la influencia de la edad en las opiniones sobre las redes sociales, el coeficiente de correlación de Spearman muestra que cuanto más jóvenes son más tendencia a pensar que estas "Permiten tener un mayor conocimiento sobre la realidad" (-.166**), pero conforme avanza la edad aumenta también el grado de acuerdo con la idea de que "Las redes sociales son clave para poder tomar decisiones en asuntos sociales y políticos" (.102*).

En relación con la clase social, el gamma de Goodman-Kruskal $(\gamma)$ nos muestra una asociación positiva significativa con dos de las opiniones analizadas: "La gente miente mucho en redes sociales" ( $\gamma<-.221, p<0.01) ; y$ "Hay que tener mucho cuidado con la información personal que se sube a redes sociales" $(\gamma<-.167, p<0.01)$. Aplicando el test de Kruskal-Wallis, se observó que la clase social mostró un efecto significativo sobre dos de las afirmaciones en $\chi^{2}(4, N=520 ; p<0.01 * *$; y $\left.p<.05^{*}\right)$, aunque una no coincide con la que nos indicó el test anterior:

- La gente miente mucho en redes sociales (estadístico de prueba $=22,51^{* *}, \eta^{2}=.040$ ). Con diferencias significativas entre clase alta y media-media $(p<.05)$ y clase media-baja $(p<.01)$; y clase media-alta y clase media-baja $(p<0.01)$ (test post-hoc con corrección Bonferroni).

- Las redes sociales facilitan que la gente corriente pueda defender sus intereses (estadístico de prueba= $\left.10,62^{*} ; \eta^{2}=.017\right)$ con diferencias significativas entre clase baja y media-alta $(p<.05)$; y clase alta y media-alta $(p<0.01)$ y clase media-baja y clase media-alta $(p<.05)$; clase media-media y media-alta $(p<.05)$ (test Los jóvenes creen que los comentarios que las personas hacen en las redes sociales no tienen una capacidad transformadora en lo político o social post-hoc con corrección Bonferroni). 
Eta al cuadrado $\left(\eta^{2}\right)$ para el test de Kruskal-Wallis nos indica que el tamaño del efecto de la clase social sobre el grado de acuerdo con las anteriores afirmaciones es moderado y débil, respectivamente; con una tendencia de las clases más bajas a pensar que la gente miente en redes sociales.
Los jóvenes manifiestan un cierto temor al juicio público al que pueden someterse sus opiniones cuando son expresadas en un foro como los medios sociales

\subsection{Opiniones que explican los tipos de uso de redes sociales por los jóvenes}

Finalmente, en este apartado se explora si las representaciones de los jóvenes sobre las redes sociales influyen en el tipo de utilización. Partiendo de nuevo de las puntuaciones resumidas en el análisis factorial que identifica cuatro modalidades de usos de redes sociales, se realizó un análisis de correlaciones (coeficiente de correlación de Spearman) con las diferentes opiniones sobre redes sociales, que mostró diferencias significativas, en particular para los usos participativos y de sociabilidad cercana; sin embargo, el grado de asociación oscila entre mínimo $(<0.2)$ a débil (entre 0.2 y 0.4 ) (véase tabla 5). Nos fijaremos, por tanto, sólo en aquellos items que manifiesten un grado de asociación igual o mayor de 0.2.

Tabla 5. Correlaciones entre tipos de usos de redes sociales y opiniones sobre redes sociales (rho de Spearman)

\begin{tabular}{|c|c|c|c|c|}
\hline & $\begin{array}{l}\text { Usos } \\
\text { informativos }\end{array}$ & $\begin{array}{l}\text { Usos } \\
\text { participativos }\end{array}$ & $\begin{array}{l}\text { Sociabilidad } \\
\text { cercana }\end{array}$ & $\begin{array}{l}\text { Usos } \\
\text { recreativos }\end{array}$ \\
\hline Las redes sociales favorecen que todos estemos mejor informados & .037 & $.117^{* *}$ & $.281^{* *}$ & -.005 \\
\hline $\begin{array}{l}\text { A través de las redes la gente puede compartir sus opiniones y experiencias } \\
\text { con la comunidad }\end{array}$ & .072 & .002 & $.185^{* *}$ & .029 \\
\hline La gente solo muestra su cara más favorable en las redes sociales & -.025 & .026 & $.187^{* *}$ & -.064 \\
\hline $\begin{array}{l}\text { Hay que tener mucho cuidado con las opiniones que se suben a las redes } \\
\text { sociales porque puede tener consecuencias negativas }\end{array}$ & -.001 & -.042 & $.161^{* *}$ & -.013 \\
\hline $\begin{array}{l}\text { Las redes sociales permiten una mayor participación de la gente corriente, } \\
\text { influyendo en las opiniones de otros }\end{array}$ & .01 & .076 & $.285^{* *}$ & -.074 \\
\hline Permiten tener un mayor conocimiento sobre la realidad que nos rodea & .019 & $.193^{* *}$ & $.270^{* *}$ & $-.090^{*}$ \\
\hline En las redes sociales cualquiera puede opinar sin miedo a tener represalias & .043 & $.169^{* *}$ & $.220^{* *}$ & -.052 \\
\hline La gente miente mucho en redes sociales & .012 & -.062 & $.214^{* *}$ & -.085 \\
\hline Hoy en día estás totalmente controlado en las redes sociales & .035 & $.086^{*}$ & $.207^{* *}$ & -.025 \\
\hline Las redes sociales facilitan que la gente corriente pueda defender sus intereses & -.009 & $.225^{* *}$ & $.227^{* *}$ & $-.111^{*}$ \\
\hline En redes se pueden encontrar otras ideas y puntos de vista muy interesantes & $.087^{*}$ & $.105^{*}$ & $.290^{* *}$ & -.048 \\
\hline Las redes sociales te aíslan de la vida real & .035 & $.104^{*}$ & $.171^{* *}$ & -.061 \\
\hline $\begin{array}{l}\text { Las redes sociales son clave para que podamos tomar decisiones en asuntos } \\
\text { sociales y políticos }\end{array}$ & .046 & $.250^{* *}$ & $.241^{* *}$ & -.027 \\
\hline $\begin{array}{l}\text { Las redes sociales proporcionan a la gente corriente más capacidad de influir } \\
\text { en asuntos públicos }\end{array}$ & .012 & $.214^{* *}$ & $.212^{* *}$ & .001 \\
\hline $\begin{array}{l}\text { Hay que tener mucho cuidado con la información personal que se sube a } \\
\text { redes sociales }\end{array}$ & .017 & -.072 & $.183^{* *}$ & -.065 \\
\hline La gente solo muestra su cara más favorable en las redes sociales & -.066 & $.087^{*}$ & $.213^{* *}$ & -.08 \\
\hline $\begin{array}{l}\text { Las redes sociales hacen posible que todos podamos participar en el debate } \\
\text { sobre temas públicos }\end{array}$ & -.006 & $.158^{* *}$ & $.200^{* *}$ & -.032 \\
\hline $\begin{array}{l}\text { Los comentarios que la gente corriente sube a las redes sociales no sirven } \\
\text { para cambiar nada }\end{array}$ & -.057 & $.227^{* *}$ & $.171^{* *}$ & -.07 \\
\hline $\begin{array}{l}\text { Las redes sociales son herramientas controladas por intereses económicos y/o } \\
\text { políticos }\end{array}$ & -.052 & $.174^{* *}$ & $.240^{* *}$ & $-.122^{* *}$ \\
\hline $\begin{array}{l}\text { Las redes sociales permiten que la gente pueda mostrar sus quejas o malestar } \\
\text { sobre las cosas que no les gusta }\end{array}$ & .013 & $.137^{* *}$ & $.337^{* *}$ & $-.112^{*}$ \\
\hline
\end{tabular}

** Diferencias significativas para $p<.01$

* diferencias significativas $p<.05$

Los usos participativos se asocian positivamente con opiniones relativas a la posibilidad de tomar decisiones en temas sociales y políticos, facilitar que la gente corriente pueda defender sus intereses, influir sobre asuntos públicos; no obstante, cabe destacar que, contrariamente a lo esperado y con una gran distancia con respecto a su grado de asociación a los usos de sociabilidad cercana, también se asocian con la creencia de que los comentarios que se suben a las redes sociales no sirven para cambiar nada. 


\section{Conclusiones}

El estudio pone de manifiesto que la práctica totalidad de los jóvenes acceden a las redes sociales a diario y su consulta es frecuente, de modo que se puede calificar su uso como intensivo. Se trata de una tendencia en aumento que se consolida a nivel global, tanto en lo referido al número de usuarios en las redes sociales como al tiempo que se dedica a las mismas (Statista, 2019). La presente investigación permite concluir que en los medios sociales los jóvenes optan de manera preferencial por conversar con sus amigos y familiares, un factor asociado al interés de los jóvenes por conocer lo que sucede en su entorno más cercano, una preocupación corroborada por el estudio de Pastor-Ruiz, Martín-Nieto y Montes-Vozmediano (2019). Las redes sociales son para los jóvenes un medio que pueden emplear con fines lúdicos y de entretenimiento, un hallazgo en sintonía con lo concluido por López-González y Anaya-Rodríguez (2016).

Por otra parte, se ha confirmado que usos más participativos, como publicar opiniones sobre temas sociales o políticos y emitir críticas o quejas en un perfil público, no forman parte de las preferencias juveniles pues son actividades apenas realizadas por una quinta parte de los encuestados. Esta evidencia pone de manifiesto el contraste que existe entre la valoración que se realiza de las redes sociales como una vía que permite a cualquier individuo expresar sus opiniones (sobre temas sociales, políticos o de otra índole) en un foro público cuyo alcance potencial es muy amplio y la consideración que se tiene sobre la escasa capacidad de cambio que se atribuye a la publicación de estos comentarios. Los jóvenes creen que los comentarios de las personas comunes en las redes sociales no tienen una capacidad transformadora en ámbitos de relevancia como lo político o social.

Sin embargo, cuando se trata de asuntos más concretos, sí se otorga de mayor consideración al efecto que pueden lograr los comentarios publicados en las redes. Los jóvenes consideran que las quejas o halagos hacia un determinado servicio, producto, evento o similar, es decir lo que se expresa en relación a un hecho específico sí es tenido en cuenta por otros usuarios y consideran que esas opiniones pueden influir en las decisiones o acciones de otras personas.

Los jóvenes también manifiestan un cierto temor al juicio público al que pueden someterse sus opiniones cuando son expresadas en un foro como los medios sociales, pues las consecuencias de exponerse pueden derivar en resultados imprevisibles.

Variables como la edad, el sexo y la clase social condicionan las valoraciones realizadas sobre las redes sociales, siendo los chicos y los jóvenes pertenecientes a clases más altas los que manifiestan valoraciones más positivas respecto a estos canales de comunicación, en su calidad de vehículos de participación y de generación de influencia social, si bien estas apreciaciones van asociadas a un uso cauteloso o precavido. Estas variables sociodemográficas también inciden en los tipos de usos en los medios sociales, que se han concretado en cuatro modalidades de uso: informativo, participativo, sociabilidad cercana y recreativo, en sintonía con lo expresado por Masip et al. (2015), si bien relegando la vertiente profesional (lo cual era previsible a tenor del público joven analizado) por la participativa.

Desde las clases media alta y alta es más frecuente el modelo de uso participativo, con una influencia casi insignificante sobre los usos de sociabilidad cercana orientada a usos de ocio audiovisual e interacción con amigos y familiares. Desde una perspectiva de género las mayores divergencias entre hombres y mujeres se concentran en los usos recreativos vinculados al juego online (Golpe et al., 2017) y el deporte, preferidos por los jóvenes frente a los modelos de usos de sociabilidad cercana e informativos que son adoptados por sus homónimas mujeres (Pastor-Ruiz; Martín-Nieto; Montes-Vozmediano, 2019), aunque las diferencias en estos últimos no son muy acentuadas. Tomando como referencia la edad del joven, a menor edad se incrementa la tendencia a usar las redes sociales para usos de sociabilidad cercana y en menor medida participativos.

\section{Nota}

1. Una posible limitación que debe tenerse en cuenta es que este estudio se ha llevado a cabo exclusivamente en la Comunidad de Madrid. Esta Comunidad uniprovincial es una de las diecisiete comunidades autónomas que conforman el Estado Español, y aloja la capital de España, Madrid. Su población estimada a 1 de enero de 2019 es 6.641 .648 de habitantes (INE, 2020), lo que significa un 14,15\% del conjunto de la población residente en España.

\section{Referencias}

Awoleye, O. Michael; Siyanbola, W. Owolabi; Oladipo, O. Francisca (2008). "Adoption assessment of Internet usage amongst undergraduates in Nigeria universities. A case study approach". Journal of technology management \& innovation, v. 3, n. 1, pp. 84-89.

https://www.jotmi.org/index.php/GT/article/view/cas11/441 
Birba, Ousmane; Diagne, Abdoulaye (2012). "Determinants of adoption of Internet in Africa: Case of 17 sub-Saharan countries". Structural change and economic dynamics, v. 23, n. 4, pp. 463-472.

https://doi.org/10.1016/j.strueco.2012.06.003

Blank, Grant; Groselj, Darja (2014). "Dimensions of internet use: Amount, variety, and types". Information communication \& society, v. 17, n. 4, pp. 417-435.

https://doi.org/10.1080/1369118x.2014. 889189

Borg, Kim; Smith, Liam (2018). "Digital inclusion and online behaviour: five typologies of Australian internet users". Behaviour \& information technology, v. 37, n. 4, pp. 367-380.

https://doi.org/10.1080/0144929x.2018.1436593

Brandtzæg, Peter-Bae; Heim, Jan; Karahasanović, Amela (2011). “Understanding the new digital divide - a typology of Internet users in Europe". International journal of human-computer studies, v. 69, n. 3, pp. 123-138.

https://doi.org/10.1016/j.ijhcs.2010.11.004

Büchi, Moritz; Just, Natascha; Latzer, Michael (2016). “Modeling the second-level digital divide: A five-country study of social differences in Internet use". New media \& society, v. 18, n. 11, pp. 2703-2722.

https://doi.org/10.1177/1461444815604154

Catalina-García, Beatriz; Martín-Nieto, Rebeca; López-de-Ayala, María-Cruz (2018). "Social media and the political-civic participation of young people. A review of the digital citizenship debate”. Doxa comunicación, n. 27, pp. 81-97. https://doi.org/10.31921/doxacom.n27a4

Correa, Teresa (2015). "Digital skills and social media use: how Internet skills are related to different types of Facebook use among digital natives". Information, communication \& society, v. 19, n. 8, pp. 1095-1107.

https://doi.org/10.1080/1369118x.2015.1084023

Datareportal (2020). Digital 2020: Global digital overview.

https://datareportal.com/reports/digital-2020-global-digital-overview

Drumbl, Mark A. (2012). "Child soldiers and clicktivism: Justice, myths, and prevention". Journal of human rights practice, v. 4, n. 3, pp. 481-485.

https://doi.org/10.1093/jhuman/hus023

García-Estévez, Noelia (2018). "Origin, evolution and current status of digital activism and its social commimtment. Ciberactivism, hacktivism and slacktivism". En: Candón-Mena, José (coord.). Actas del II Congreso internacional Move.net sobre movimientos sociales y TIC, pp. 139-156.

García-Jiménez, Antonio; López-de-Ayala-López, María-Cruz; Catalina-García, Beatriz (2013). "The influence of social networks on the adolescents' online practices". Comunicar, v. 21, n. 41, pp. 195204.

https://doi.org/10.3916/c41-2013-19

García-Jiménez, Antonio; López-de-Ayala-López, María-Cruz; Montes-Vozmediano, Manuel (2020). “Características y percepciones sobre el uso de las plataformas de redes sociales y dispositivos tecnológicos por parte de los adolescentes". Zer, v. 25, n. 48, pp. 269-286.

https://doi.org/10.1387/zer.21556

García-Jiménez, Antonio; Montes-Vozmediano, Manuel (2020). “Subject matter of videos for teens on YouTube”. International journal of adolescence and youth, v. 25, n. 1, pp. 63-78.

https://doi.org/10.1080/02673843.2019.1590850

Gloy, Brent A.; Akridge, Jay T. (2000). "Computer and internet adoption on large U.S. farms". The international food and agribusiness management review, v. 3, n. 3, pp. 323-338.

https://doi.org/10.1016/s1096-7508(01)00051-9

Golpe-Ferreiro, Sandra; Gómez-Salgado, Patricia; Harris, Sion-Kim; Braña-Tobío, Teresa; Rial-Boubeta, Antonio (2017). "Diferencias de sexo en el uso de Internet en adolescentes españoles". Behavioral psychology / Psicología conductual, v. 25, n. 1 , pp. $129-146$.

https://www.behavioralpsycho.com/product/gender-differences-in-the-use-of-the-internet-in-spanish-adolescents

Gonzales, Amy L.; Yan, Harry-Yaojun (2020). "Non-profit reuse as a solution to reducing digital divides and technology maintenance inequalities". In: Doerfel, Marya L.; Gibbs, Jennifer L. (eds.). Organizing inclusion. Moving diversity from demographics to communication processes. London: Routledge. ISBN: 9781138325272

Hill, Rebecca; Beynon-Davies, Paul; Williams, Michael D. (2008). "Older people and internet engagement". Information technology \& people, v. 21, n. 3, pp. 244-266.

https://doi.org/10.1108/09593840810896019 
Kaplan, Andreas M.; Haenlein, Michael (2010). "Users of the world, unite! The challenges and opportunities of social media". Business horizons, v. 53, n. 1, pp. 59-68.

https://doi.org/10.1016/j.bushor.2009.09.003

Karpf, David (2010). "Online political mobilization from the advocacy group's perspective: Looking beyond clicktivism". Policy \& internet, v. 2, n. 4, pp. 7-41.

https://goo.gl/zS6Hrf

Khalaila, Rabia; Vitman-Schorr, Adi (2018). "Internet use, social networks, loneliness, and quality of life among adults aged 50 and older: mediating and moderating effects". Quality of life research, n. 27, pp. 479-489.

https://doi.org/10.1007/s11136-017-1749-4

Kircaburun, Kagan (2016). "Self-esteem, daily internet use and social media addiction as predictors of depression among Turkish adolescents". Journal of education and practice, v. 24, n. 7, pp. 64-72.

https://files.eric.ed.gov/fulltext/EJ1112856.pdf

Knight-McCord, Jasmine; Cleary, Dylan; Grant, Nastassjia; Herron, Antoinette; Jumbo, Success; Lacey, Tiffany; Livingston, Torri; Robinson, Sky; Smith, Renardo; Emanuel, Richard (2016). "What social media sites do college students use most?". Journal of undergraduate ethnic minority psychology, n. 2, pp. 21-26.

http://www.juempsychology.com/wp-content/uploads/2016/05/Knight-McCord_et-al_JUEMP_2016.pdf

Liu, Dong; Kirschner, Paul A.; Karpinski, Aryn C. (2017). "A meta-analysis of the relationship of academic performance and social network site use among adolescents and young adults". Computers in human behavior, n. 77, pp. 148-157. https://doi.org/10.1016/j.chb.2017.08.039

López-González, Rocío; Anaya-Rodríguez, Roberto (2016). “Estudiantes universitarios interactuando en red: ¿nuevos escenarios de interacción, expresión y participación ciudadana?”. Revista interamericana de educación de adultos, v. 38, n. 1, pp. 48-67.

https://www.redalyc.org/pdf/4575/457545337004.pdf

Masip, Pere; Guallar, Javier; Suau, Jaume; Ruiz-Caballero, Carlos; Peralta, Miquel (2015). “News and social networks: Audience behaviour". El profesional de la información, v. 24, n. 4, pp. 363-370.

https://doi.org/10.3145/epi.2015.jul.02

Nasri, Wadie; Charfeddine, Lanouar (2012). "Factors affecting the adoption of Internet banking in Tunisia: An integration theory of acceptance model and theory of planned behavior". The journal of high technology management research, v. 23, n. 1, pp. 1-14.

https://doi.org/10.1016/j.hitech.2012.03.001

Nowland, Rebecca; Necka, Elizabeth A.; Cacioppo, John T. (2017). "Loneliness and social internet use: pathways to reconnection in a digital world?". Perspectives on psychological science, v. 13, n. 1, pp. 70-87.

https://doi.org/10.1177/1745691617713052

Pastor-Ruiz, Yolanda; Martín-Nieto, Rebeca; Montes-Vozmediano, Manuel (2019). “Patrones de uso, control parental y acceso a la información de los adolescentes en la red”. Estudios sobre el mensaje periodístico, v. 25, n. 2, pp. 995-1012. https://doi.org/10.5209/esmp.64821

Ragnedda, Massimo; Muschert, Glenn W. (2018). “Introduction”. In: Ragnedda, Massimo; Muschert, Glenn W. (eds.). Theorizing digital divides. London: Routledge, pp 1-7. ISBN: 9781315455334

Ros-Martín, Marcos (2009). "Evolución de los servicios de redes sociales en internet". El profesional de la Información, v. 18, n. 5 , pp. 552-557.

https://doi.org/10.3145/epi.2009.sep.10

Sánchez-Martínez, Mercedes; Otero, Ángel (2010). “Usos de internet y factores asociados en adolescentes de la Comunidad de Madrid". Atención primaria, v. 42, n. 2, pp. 79-85.

https://doi.org/10.1016/j.aprim.2009.05.004

Sathye, Milind (1999). "Adoption of Internet banking by Australian consumers: an empirical investigation". International journal of bank marketing, v. 17, n. 7, pp. 324334.

https://doi.org/10.1108/02652329910305689

Shensa, Ariel; Escobar-Viera, César G.; Sidani, Jaime E.; Bowman, Nicholas D.; Marshal, Michael P.; Primack, Brian A. (2017). "Problematic social media use and depressive symptoms among U.S. young adults: A nationally-representative study". Social science \& medicine, v. 182, pp. 150157.

https://doi.org/10.1016/j.socscimed.2017.03.061

Statista (2019). Social media usage worldwide.

https://www.statista.com/study/12393/social-networks-statista-dossier 
Symons, Katrien; Vanwesenbeeck, Ini; Walrave, Michel; Van-Ouytsel, Joris; Ponnet, Koen (2019). “Parents' concerns over internet use, their engagement in interaction restrictions, and adolescents' behavior on social networking sites". Youth \& society, n. 1, pp. 1-13.

https://doi.org/10.1177/0044118x19834769

Van-Rompaey, Veerle; Roe, Keith; Struys, Karin (2002). “Children's influence on internet access at home: Adoption and use in the family context". Information, communication \& society, v. 5, n. 2, pp. 189206.

https://doi.org/10.1080/13691180210130770

Vizcaíno-Laorga, Ricardo; Catalina-García, Beatriz; López-de-Ayala, María-Cruz (2019). “Participation and commitment of young people in the digital environment. Uses of social networks and perception of their consequences". Revista latina de comunicación social, n. 74, pp. 554-572.

http://www.revistalatinacs.org/074paper/1345/28en.html

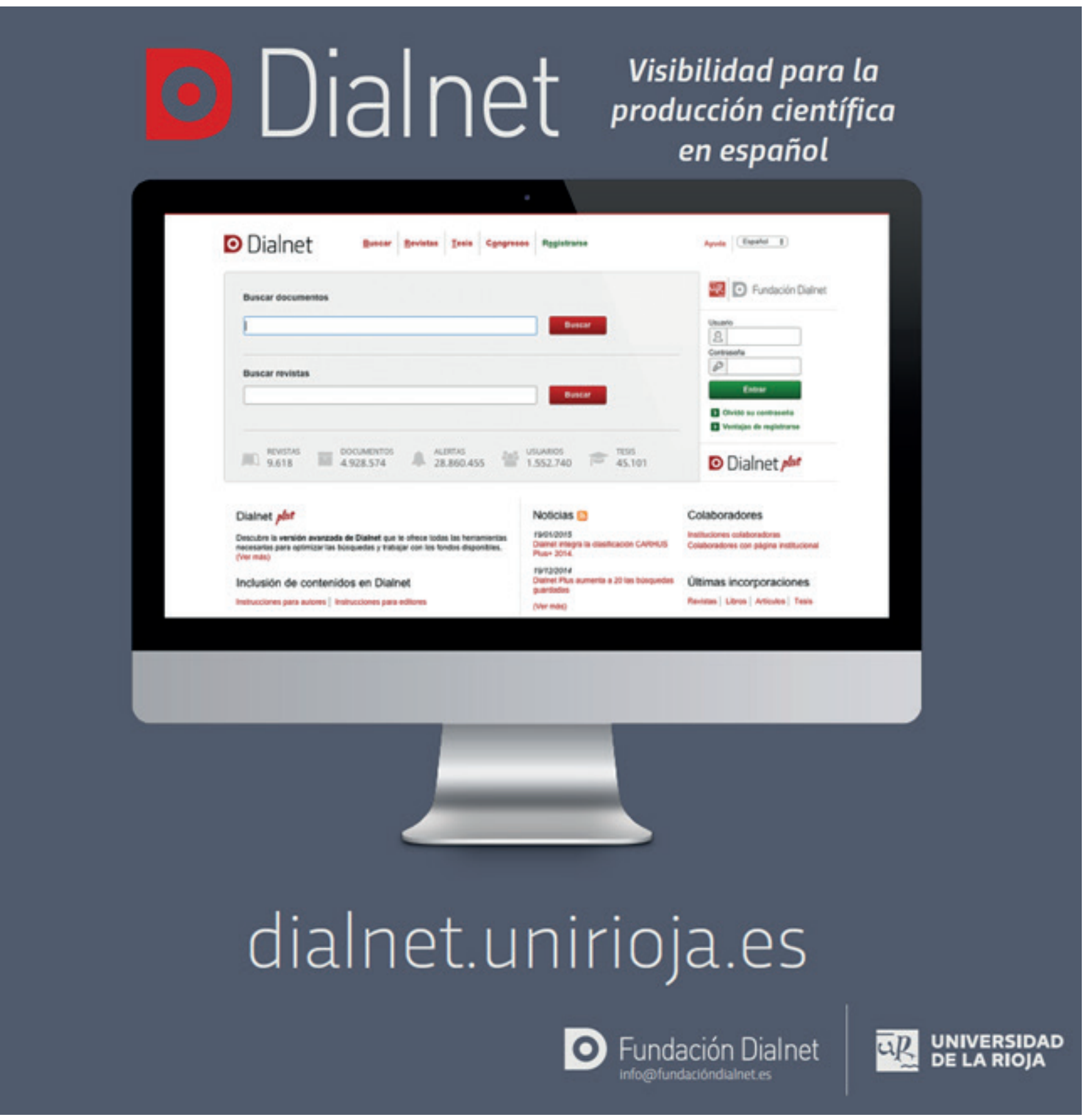

